\title{
Unlocking the therapeutic potential of primary tumor-draining lymph nodes
}

\author{
Jossie Rotman ${ }^{1}$ - Bas D. Koster ${ }^{2}$ Ekaterina S. Jordanova ${ }^{1}$ - A. Marijne Heeren ${ }^{2} \cdot$ Tanja D. de Gruijl $^{2}$
}

Received: 19 November 2018 / Accepted: 22 March 2019 / Published online: 3 April 2019

(c) The Author(s) 2019

\begin{abstract}
Lymph nodes draining the primary tumor are essential for the initiation of an effective anti-tumor T-cell immune response. However, cancer-derived immune suppressive factors render the tumor-draining lymph nodes (TDLN) immune compromised, enabling tumors to invade and metastasize. Unraveling the different mechanisms underlying this immune escape will inform therapeutic intervention strategies to halt tumor spread in early clinical stages. Here, we review our findings from translational studies in melanoma, breast, and cervical cancer and discuss clinical opportunities for local immune modulation of TDLN in each of these indications.
\end{abstract}

Keywords Tumor-draining lymph node $\cdot$ Local immunotherapy · Cervical cancer · Melanoma · Breast cancer · TIMO 2018

$\begin{array}{ll}\text { Abbreviations } \\ \text { BrC } & \text { Breast cancer } \\ \text { cDC } & \text { Conventional dendritic cell(s) } \\ \text { CxCa } & \text { Cervical cancer } \\ \text { ICD } & \text { Immunogenic cell death } \\ \text { LN- } & \text { Tumor-negative lymph node } \\ \text { LN+ } & \text { Tumor-positive lymph node } \\ \text { LNDC } & \text { Lymph node resident dendritic cell(s) } \\ \text { NAC } & \text { Neo-adjuvant chemotherapy } \\ \text { pCR } & \text { Pathologic complete response } \\ \text { pDC } & \text { Plasmacytoid dendritic cell(s) } \\ \text { PGE2 } & \text { Prostaglandin-E2 } \\ \text { SLN } & \text { Sentinel lymph node(s) } \\ \text { SNB } & \text { Sentinel node biopsy } \\ \text { TDLN } & \text { Tumor-draining lymph node(s) }\end{array}$

Tanja D. de Gruijl

TD.deGruijl@vumc.nl

1 Department of Obstetrics and Gynecology, Center for Gynecological Oncology Amsterdam (CGOA), Amsterdam UMC, Cancer Center Amsterdam, Vrije Universiteit Amsterdam, Amsterdam, The Netherlands

2 Department of Medical Oncology, Amsterdam UMC, Cancer Center Amsterdam, Vrije Universiteit Amsterdam, De Boelelaan 1117, 1081 HV Amsterdam, The Netherlands

\section{Introduction}

Many complex processes are involved in the metastatic spread of cancer cells from the primary tumor to lymph nodes and distant organs. The sentinel lymph node (SLN) is the first node to receive lymphatic drainage from the primary lesion and is of great importance in initiating an effective anti-tumor immune response; it also constitutes a first line of defense against metastatic spread [1]. For many malignancies, the presence of tumor cells in tumor-draining lymph nodes (TDLN), and in the SLN in particular, is a key prognostic factor and, in some cases, predicates the course of treatment [2]. In some tumors, e.g., cervical cancer (CxCa) or oral cancer, a complete lymphadenectomy provides overall survival benefit [3-6]. However, for other indications, such as melanoma [7] and breast cancer ( $\mathrm{BrC})$, this is not the case [8].

\section{Tumor-draining lymph nodes as a target for immunotherapy}

The main focus of current immunotherapeutic strategies is on targeting the microenvironment of primary tumors and/ or metastatic lesions, most notably by checkpoint inhibitors. As therapeutic targets, TDLN, and SLN in particular, are relatively undervalued, and clinically under-utilized. They are, nonetheless, essential players in anti-tumor immunity. In this focused review, we will discuss the importance of, and clinical opportunities for, therapeutic targeting of TDLN, 
based on findings from pre-clinical and clinical studies carried out by our group.

In the TDLN, tumor-specific T-cell responses are initiated. Here, effective priming of cytotoxic $\mathrm{CD} 8^{+} \mathrm{T}$ cells takes place upon tumor-specific (neo)antigen recognition, presented by APC, including DC and macrophages [9]. Although DC represent only a small population of all the immune cell subsets in the LN, they are crucial in initiating an effective immune response. In cancer, however, TDLN are under the influence of tumor-derived factors, such as extracellular vesicles [1], IL-6 [10], TGF- $\beta$ [11], prostaglandin-E2 (PGE2) [10], and VEGF [12, 13]. As a result, DC are suppressed and acquire an immature and M2 macrophage-like phenotype, and will, therefore, not properly cross-present in TDLN [14]. During tumor progression and prior to metastasis, TDLN undergo many additional profound alterations leading to invasion by cells derived from the primary tumor $[1,2,15]$. Such alterations include increased lymphangiogenesis, blood vessel remodeling, and increased chemokine and cytokine secretion, which can ultimately lead to changes in immune cell composition, resulting in a 'tumor-supportive' microenvironment, i.e., the pre-metastatic niche [1]. Moreover, with the ability of tumor cells to evade immune surveillance by the upregulation of immunosuppressive ligands and downregulation of MHC class I-molecules, this can eventually lead to the metastatic growth of tumor cells that have reached the TDLN [1].

Thus, immune modulation of TDLN could generate effective tumor-specific T-cell responses and in this way prevent metastatic spread. Considering that only a minor fraction of systemically administered drugs reaches the TDLN [16], locally applied therapies may be more effective in counteracting immune suppression in TDLN. Based on immune profiling and ex vivo proof-of-concept studies, we have conducted and are currently conducting a number of clinical trials aimed at immune potentiation of the TDLN through local delivery of immune modulatory drugs.

\section{Immune profiling of lymph nodes in cancer}

Over the past 2 decades, our group has pioneered the flow cytometry-based immune profiling of TDLN in humans. In these studies, we employ a scraping method (i.e., we scrape the cutting surface of a bisected TDLN) to obtain viable leukocytes from the TDLN, which was shown not to interfere with diagnostic procedures [17]. Compared to dissociation of the entire node, we found similar viabilities and phenotypic characteristics of T-cell and DC subsets in scrapes [18]. In addition, using multiparameter (fluorescent) IHC, we are currently working on improving our understanding of the TDLN architecture and cellular networks by studying (co-)localization of diverse immune cell subsets in their microenvironment [19-21].

\section{The influence of primary and invasive melanoma on conventional DC in SLN}

In early pioneering studies on the immune status of melanoma SLN, Cochran and colleagues convincingly demonstrated that DC in SLN were more immune suppressed than DC in further downstream located TDLN [22, 23]. This observation suggested DC to be a prime target of melanomainduced immune suppression, consistent with their pivotal role in initiating T-cell-mediated anti-tumor immunity. Our group was the first to characterize and functionally test conventional DC (cDC) subsets, distinguishing migratory from LN-resident (LNDC) subsets, in human skin-draining LN using multiparameter flow cytometry [24] closely followed by Segura and colleagues [25]. We identified two migratory $\mathrm{CD} \mathrm{a}^{+} \mathrm{cDC}$ subsets, i.e., dermal DC (DDC) and Langerhans cells (LC), and two $\mathrm{LNDC} \mathrm{CD1a}^{-} \mathrm{cDC}$ subsets, distinguished by absence or presence of CD14 expression (see Table 1). The relative importance and varying roles of these $\mathrm{cDC}$ subsets in the priming of immune responses in healthy human LN remains largely elusive, but some clues are emerging. The migratory subsets take up antigens in the skin and will subsequently migrate to the skin-draining $\mathrm{LN}$, where they can present those antigens to $\mathrm{T}$ cells. The two LNDC subsets are found in skin-draining LN but not among DC migrated from skin explants and are recruited from the peripheral blood to the LN [26]. They are key players in cross presentation as evidenced by the high surface levels of cross-priming associated markers CLEC9A and BDCA3/CD141 (as well as expression of BATF3 mRNA; van de Ven et al., unpublished data) and by correlation of their frequencies to cross-presentation ability of melanoma SLN single-cell suspensions, which we observed after TLR9-mediated conditioning [26]. Importantly, although the migratory subsets appeared more phenotypically mature under steady-state conditions, ex vivo isolated LNDC (both
Table 1 Conventional dendritic cell subsets found in skindraining lymph nodes

\begin{tabular}{llll}
\hline Name & Phenotype [24] & Origin [24] & Most affected by [27] \\
\hline Langerhans cells & $\mathrm{CD} 1 \mathrm{a}^{\text {hi }} \mathrm{CD} 11 \mathrm{c}^{\text {int }}$ & Skin (migratory) & Primary tumor \\
Dermal dendritic cells & $\mathrm{CD} 1 \mathrm{a}^{\text {int }} \mathrm{CD} 11 \mathrm{c}^{\text {hi }}$ & Skin (migratory) & Primary tumor \\
$\mathrm{CD} 14^{-}$LNDC & $\mathrm{CD} 1 \mathrm{a}^{-} \mathrm{CD} 11 \mathrm{c}^{+} \mathrm{BDCA}^{\text {hi }} \mathrm{CD} 14^{-}$ & Circulation (LN resident) & LN metastasis \\
$\mathrm{CD} 14^{+}$LNDC & $\mathrm{CD} 1 \mathrm{a}^{-} \mathrm{CD} 11 \mathrm{c}^{+} \mathrm{BDCA}^{\text {lo }} \mathrm{CD} 14^{+}$ & Circulation (LN resident) & LN metastasis \\
\hline
\end{tabular}


$\mathrm{CD}^{-} 4^{-}$and $\mathrm{CD} 14^{+}$) subsets proved more powerful in vitro primers of allogeneic effector T cells, which might tie in with higher release levels of T-cell-activating cytokines [24]. Functional differentiation between the $\mathrm{CD} 14^{-}$and $\mathrm{CD} 14^{+}$ subsets remains obscure, but both may be involved in the priming of systemic anti-tumor effector T-cell responses, as we found the activation state of either to be associated with distant recurrence-free survival in early stage melanoma [27]. Another DC subset in skin-draining LN are plasmacytoid DC (pDC) [26]. These cells are poor antigen presenters, but are powerful producers of type I interferons upon TLR activation [28]. As such, pDC play an important role in the activation of cDC and other immune cells.

In melanoma, we observed a significant negative correlation between the activation state (based on CD83 expression) of DDC and LC in the SLN and primary tumor burden (Breslow thickness) [27]. Interestingly, primary tumor burden was not shown to have a significant effect on either the frequency or activation state of LNDC subsets. However, the presence of SLN tumor metastases did have a significant impact on both the frequency and activation state of conventional LNDC, the latter showing a reverse correlation with the size of the metastasis (Table 1). This suggests that the primary melanoma can create a pre-metastatic niche in the TDLN by suppressing the activation states of migratory $\mathrm{cDC}$ subsets, which was shown to be associated with a shorter local recurrence-free survival. Subsequently, TDLN metastasis suppress LNDC which, interestingly, was shown to be associated with a worse distant recurrence-free survival [27]. The latter indicates an essential role for conventional LNDC in the induction of effective systemic anti-tumor immunity.

\section{Immune modulation of the melanoma SLN}

The 10-year melanoma-specific survival of stage I and II melanoma patients, defined as any primary tumor without regional or distant metastases, ranges from 98 to $75 \%$ depending on risk factors, such as Breslow tumor depth and tumor ulceration. After tumor spread to the regional $\mathrm{LN}$, the 10-year melanoma-specific survival can drop to as low as $24 \%$ in patients with stage IIID melanoma [29]. The unmet medical need for many of these patients stems from the fact that there is no widely used adjuvant treatment available to reduce the chances of disease recurrence, although systemic treatment (neo-adjuvant, i.e., preceding complete lymph node dissection) with immune checkpoint inhibitors in patients who are at very high risk of recurrence (high-risk stage III) and treatment with dual BRAF and MEK inhibitors in patients with BRAF V600E or V600K mutated stage III melanoma, has shown to improve recurrence-free survival [30-33], and has recently been approved by the FDA. For all other early stage patients, there is a "wait and see" approach after surgical removal of the primary lesion and SLN
Interestingly, we were able to show in multiple randomized and placebo-controlled clinical trials that there is a good rationale to treat these early stage melanoma patients with local immunotherapy aiming to prevent loco-regional and, eventually, distant spread, while minimizing immunerelated side effects in this essentially healthy population. Our earliest results were published in 2004 and reported on a 2-armed (1:1) randomized placebo-controlled phase II trial in which 12 patients received four daily intradermal injections directly adjacent to the scar from the primary melanoma excision from day -3 to day 0 , just before the sentinel node biopsy (SNB) and re-excision of the (former) primary tumor site. Patients received either $3 \mu \mathrm{g} / \mathrm{kg}$ body weight recombinant human GM-CSF dissolved in $1 \mathrm{~mL}$ saline or $1 \mathrm{~mL}$ plain saline alone. GM-CSF administration resulted in higher frequencies and enhanced maturation and activation state of CD1a ${ }^{+}$migratory cDC in the SLN [34]. In two more recent trials, we showed that low-dose intradermal injections with the TLR9 agonist CpG-B, either alone or combined with GM-CSF, at 1 week prior to the SNB, resulted in enhanced activation of conventional $\mathrm{CD} 14^{-}$and $\mathrm{CD} 14^{+}$ LNDC as well as of pDC in the SLN [26, 35]. Interestingly, this local immunotherapy instigated local (i.e., in the SLN) as well as systemic tumor-specific $\mathrm{CD} 8^{+} \mathrm{T}$-cell reactivity [36]. A recent meta-analysis showed that patients in the treatment arm of these two studies had fewer tumor-positive SLN after SNB and a longer recurrence-free survival [37]. These studies thus deliver an important proof-of-concept, showing that local immune modulation, specifically of TDLN, may lead to systemic protection against later tumor recurrences (see Fig. 1). We are currently planning a confirmatory randomized and placebo-controlled phase II clinical trial with a next-generation $\mathrm{CpG}$ oligodeoxynucleotide in 214 patients with stage II melanoma (Netherlands Trial Registry no. NTR7355).

\section{Immune modulation of TDLN in breast cancer}

Comparable DC-targeting therapeutic approaches may be implemented in patients with $\mathrm{BrC}$, since both melanoma and $\mathrm{BrC}$ drain to $\mathrm{LN}$ in the skin catchment area with comparable migratory and LN-resident DC subset distribution profiles. In $\mathrm{BrC}$, neoadjuvant chemotherapy (NAC) is one of the treatment options. A pathologic complete response (pCR) upon NAC is an independent predictor for favorable clinical outcome in all molecular subtypes [38]. Interestingly, T-cell infiltration in $\mathrm{BrC}$ holds predictive value for response to chemotherapy [39]. Since certain cytostatic drugs can induce immunogenic cell death (ICD), leading to the release of tumor-associated antigens [40], there is a clear rationale to combine NAC with DC-potentiating strategies to optimize tumor-specific T-cell priming in the TDLN. An early study from 1999 already showed a favorable effect on 


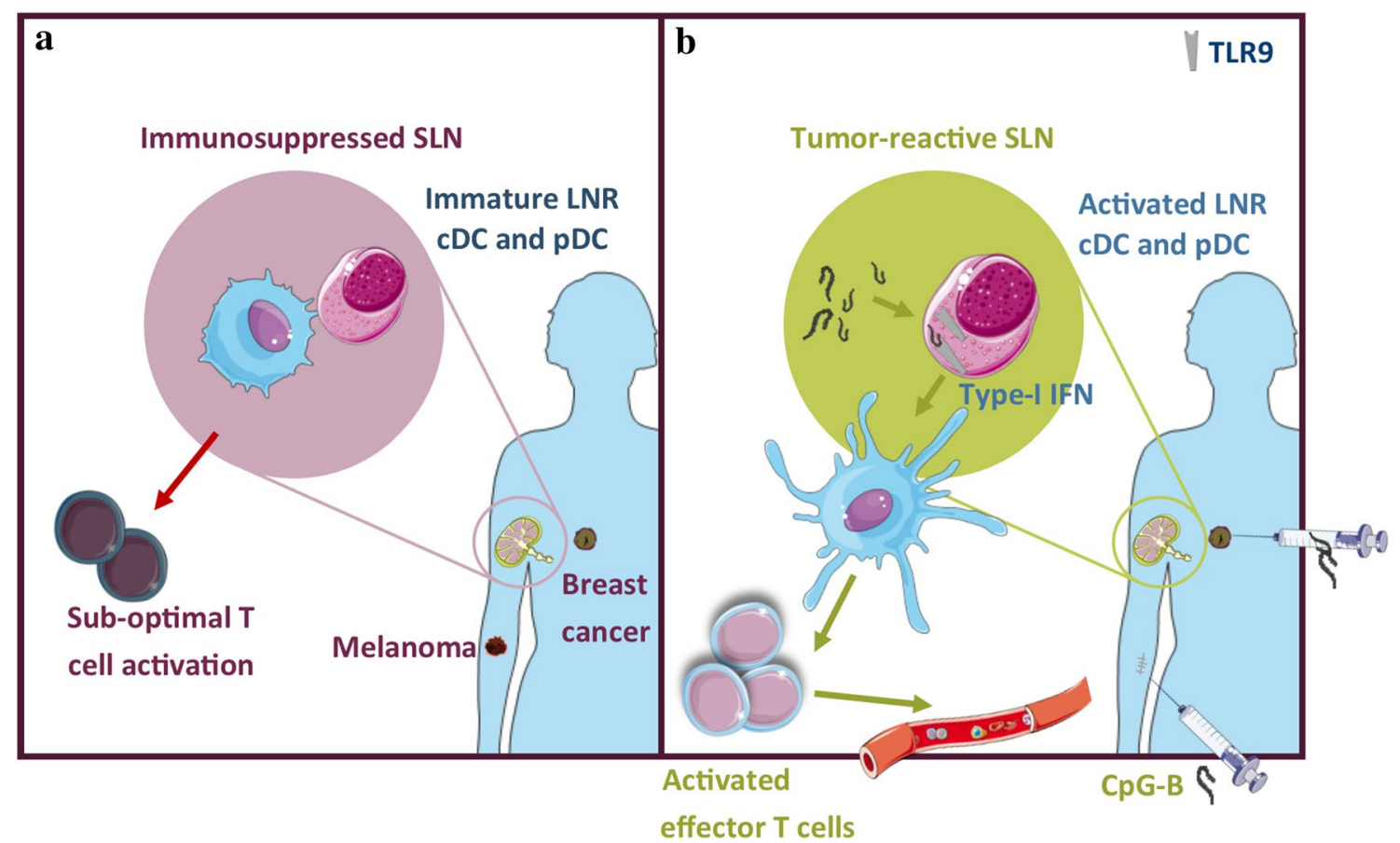

Fig. 1 In melanoma and breast cancer draining SLN, tumors can effectively suppress the activation state of LN-resident cDC (a). Immune modulation of the SLN by local injection of TLR-9 agonist

patient survival of combined GM-CSF with NAC in patients with locally advanced $\mathrm{BrC}$ [41]. Patients were treated with doxorubicin, cyclophosphamide (both agents known to induce ICD) and GM-CSF at three-weekly intervals. After a maximum of six cycles, patients underwent surgery and postoperative radiotherapy. We observed higher frequencies of mature DCs in the TDLN of these patients, suggesting that GM-CSF is able to improve patient outcome via DC recruitment and maturation, and a subsequent anti-tumor response [42]. Interestingly, we have observed a similar relationship between hampered activation of LNDC and tumor involvement of SLN in patients with $\mathrm{BrC}$ as we previously reported in melanoma (van Pul et al. manuscript submitted). Therefore, in analogy to our clinical findings in melanoma, CpG-based local immune potentiation in combination with NAC may improve response rates in patients with $\mathrm{BrC}$. This certainly deserves further (pre-)clinical exploration.

\section{The role of TDLN in cervical cancer}

In contrast to melanoma and $\mathrm{BrC}, \mathrm{CxCa}$ is caused by a persistent infection with high-risk strains of the human papillomavirus (HPV), mainly HPV16 and HPV18. HPVspecific $\mathrm{T}$ cells [43] as well as $\mathrm{T}$ cells that target non-viral tumor-associated (neo-)antigens [44] have been detected in $\mathrm{CxCa}$ TDLN. As HPV-derived antigens are highly immunogenic, it is assumed that an immunosuppressive environment
CpG-B results in activation of LN-resident $\mathrm{cDC}$ subsets (through type-1 IFN release by pDC), which ultimately leads to systemic protection against later tumor recurrences $(\mathbf{b})$

facilitates immune escape and thereby causes lymphatic spread.

$\mathrm{CxCa}$ is a locally invading disease and initially metastasizes to pelvic TDLN. The presence of LN metastases in patients with $\mathrm{CxCa}$ is a crucial prognostic factor [45]. Importantly, survival benefit was observed for $\mathrm{CxCa}$ patients who underwent complete lymphadenectomy upon low-volume disease detection in the SLN, or even upon the removal of solely tumor-negative $\mathrm{LN}[3,5]$, indicating the presence of an unfavorable immune microenvironment in $\mathrm{CxCa}$ draining pelvic LN. To understand the cellular basis for this phenomenon and to find new immunotherapeutic targets that would allow immune stimulatory conversion of the TDLN microenvironment, we performed several studies in which we found various immune escape mechanisms exploited by $\mathrm{CxCa}$.

\section{The influence of PD-L1+ M2-like macrophages on cervical cancer progression}

Interestingly, flow cytometric characterization of diverse immune cell subsets in TDLN of CxCa patients, showed that in contrast to melanoma and $\mathrm{BrC}$, Langerhans cells were hardly present in $\mathrm{CxCa} \mathrm{LN}$. Although higher levels of $\mathrm{CD}_{1} \mathrm{a}^{+} \mathrm{DCs}$ were present in tumor-positive $\mathrm{LN}(\mathrm{LN}+)$ as compared to tumor-negative $\mathrm{LN}(\mathrm{LN}-)$ [46], these cells might have been derived from recruited and tumor-converted 
monocytes rather than conventional migratory $\mathrm{CD}_{1 \mathrm{a}}{ }^{+} \mathrm{DC}$. Remarkably, we did not find evidence of decreased LNDC activation. These results point to the requirement for a different immunotherapeutic approach aimed at TDLN conditioning in $\mathrm{CxCa}$, than the one tested and proposed for melanoma and $\mathrm{BrC}$, respectively.

In addition to higher levels of $\mathrm{CD}^{+} \mathrm{a}^{+} \mathrm{DCs}$, elevated levels of activated $\mathrm{CD}^{+} \mathrm{T}$ cells in $\mathrm{LN}+$ suggested immune activation [46]. However, this activation was apparently overruled by a highly immunosuppressed microenvironment in $\mathrm{LN}+$ compared to $\mathrm{LN}-$, with high expression levels of the checkpoint molecules PD- 1 and CTLA- 4 on T cells and the presence of MDSC. Moreover, high rates of Tregs were observed in $\mathrm{LN}+$, which correlated with the rates of M2-like CD14 ${ }^{+} \mathrm{PD}-\mathrm{L}^{+}{ }^{+}$APC. A cytokine release profile consistent with an immune suppressive microenvironment was observed as well, with high IL-10, IL-6, TNF $\alpha$, and low IFN $\gamma$ expression. In a comparative study of all dissected cervical TDLN from five patients with $\mathrm{CxCa}$, we found that immune suppression (identified as low $\mathrm{CD}^{+} \mathrm{T}$ cell//FoxP3 ${ }^{+}$ Treg ratios) preceded actual metastasis, creating metastatic niches in the tumor-draining lymphatic catchment area [21]. We hypothesize that primary tumors are able to recruit (possibly via the secretion of CCL2) [47] and polarize CD14 ${ }^{+}$ monocytes into suppressive $\mathrm{PD}-\mathrm{L}^{+}{ }^{+} \mathrm{M} 2$-like macrophages [(co)-expressing CD14 and/or CD163] [48]. These M2-macrophage-like cells, induced by tumor-derived factors, are incapable of stimulating proper $\mathrm{CD} 8^{+} \mathrm{T}$-cell responses, favor Treg expansion, and facilitate tumor progression by the production of pro-angiogenic and pro-tumor-invasive factors $[14,49]$.

In aggregate, our findings support the clinical exploration of immunotherapies in $\mathrm{CxCa}$ aimed at converting the prevailing immunosuppressive conditions in the primary tumor and TDLN into an immune-activated tumor-targeting environment.

\section{Modulating TDLN in cervical cancer}

Recently, an immune checkpoint inhibitor of PD-1, pembrolizumab, was approved by the FDA for patients with recurrent or advanced $\mathrm{CxCa}$ based on an overall response rate of $14.3 \%$ and a complete response rate of (only) $2.6 \%$ [50]. Based on these results, and the fact that $\mathrm{CxCa}$ is mainly a locally invasive disease, we believe that intratumorally administered immunotherapies in earlier stages of $\mathrm{CxCa}$ may accomplish tumor control, as TDLN and the $\mathrm{PD}-\mathrm{L}^{+}{ }^{+}$macrophages residing therein are most efficiently targeted in this manner. We hypothesize that interference in the functionality of M2-like macrophages in the TDLN may hamper Treg expansion and break the vicious cycle of metastatic niche formation and tumor spread through the lymphatic catchment area, and subsequently to more distant sites (see Fig. 2). Currently, a phase-I clinical trial is ongoing, testing the safety and feasibility of a single low dose of intratumorally injected durvalumab (anti-PD-L1) in $\mathrm{CxCa}$ patients 2 weeks before radical hysterectomy with pelvic LN dissection (Netherlands Trial Registry no. NTR6119). With this strategy, we aim to achieve modulation of the microenvironment in the primary tumor and the TDLN and so break immune suppression. This will hopefully result in the generation of both local and systemic tumor-specific T-cell reactivity [51], like we previously observed when investigating locally administered CpG-B in melanoma patients, with an even shorter time window of 1 week between drug administration and surgery.

\section{Conclusion}

Immune profiling of TDLN in patients with various types of solid tumors enabled us to ascertain the suppressive effects of the tumor on loco-regional cellular immunity and provided a clear rationale for the local application of immune modulating therapies targeting TDLN (see Table 2). It is essential to perform immune profiling for each tumor type and subsequently select the appropriate immune modulating agent, as various possible mechanisms of immune suppression were found per tumor type. Importantly, we found evidence of systemic anti-tumor immune activation which seemed capable of preventing (distant) recurrences, as shown by a profoundly increased 10 -year recurrence-free survival rate in melanoma patients treated locally with $\mathrm{CpG}-\mathrm{B}$ prior to the standard-of-care SLN procedure. With the use of less invasive locally applied therapies, surgical complications resulting from $\mathrm{LN}$ dissection (e.g., lymphedema) may be avoided. Moreover, this localized therapeutic approach may stop cancer spread in its tracks at an early stage and trigger a protective systemic anti-tumor T-cell response without the unwanted, and sometimes severe, side effects associated with systemic treatment with immune checkpoint blockade [52, 53]. This may have a major impact on patient survival and quality of life. Moreover, by administering a single low dose, the high costs associated with systemic immunotherapeutic treatments in more advanced stages of cancer could be conceivably reduced [54].

In conclusion, we believe that TDLN are of major importance in initiating a robust anti-tumor response upon immune modulating therapies and should be targeted by local delivery of immune modulatory agents. Evidence for this was provided by i.t. delivery of CTLA-4 blocking antibodies in a mouse model, showing equivalent tumor control to systemic administration with reduced side effects [55]. Interestingly, Chamoto and colleagues observed absent anti-tumor efficacy of PD-1 blockade in a mouse model with TDLN ablation, and so demonstrated TDLN to be indispensable, even 


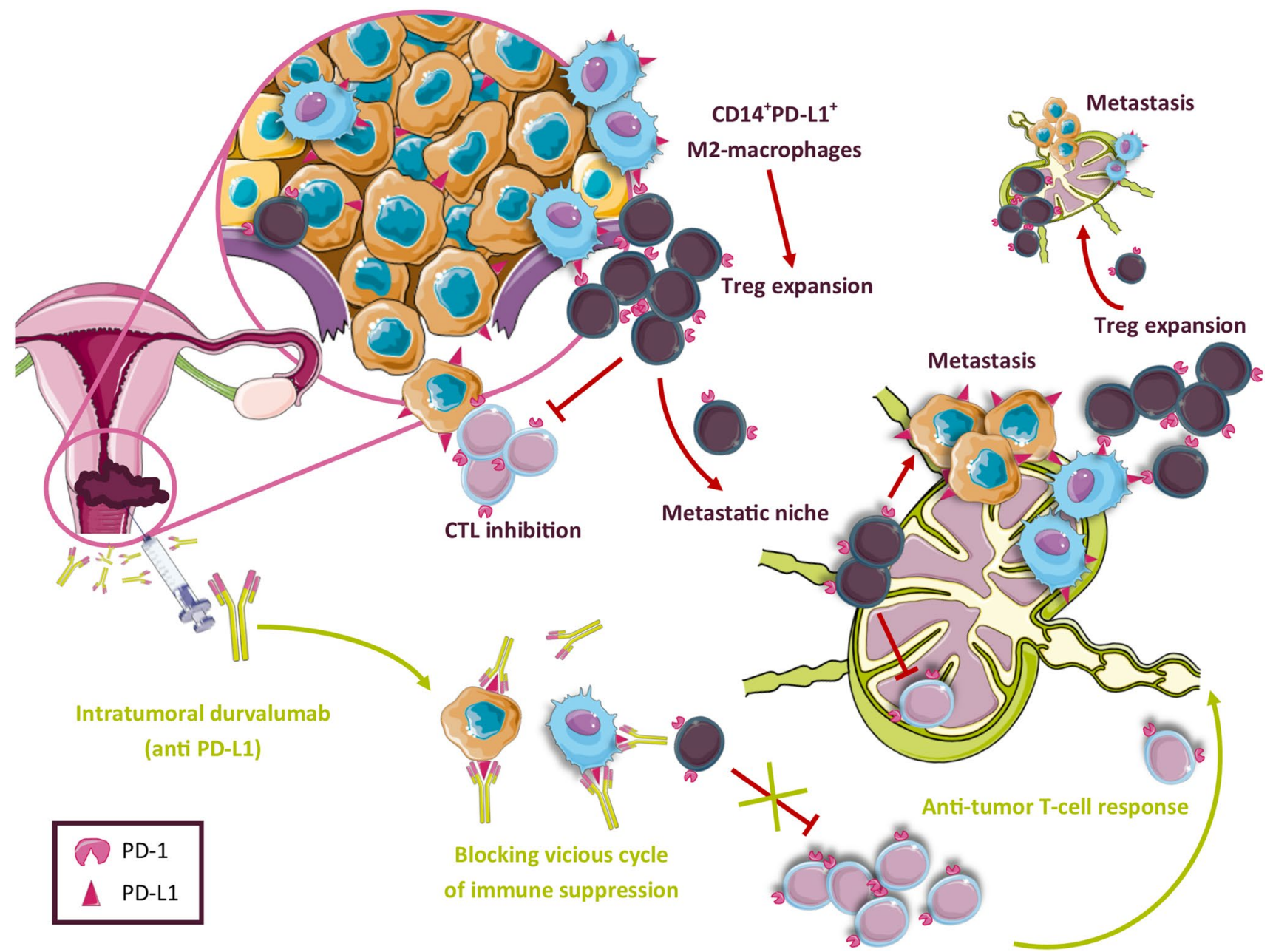

Fig. 2 Model of tumor-related immune suppression in CxCa TDLN. In the primary tumor expansion and activation of Tregs takes place through their interaction with $\mathrm{CD} 14^{+} \mathrm{PD}-\mathrm{L}^{+}{ }^{+} \mathrm{M} 2$-like macrophages (differentiated from monocytes recruited to the TME from peripheral blood). This leads to effector T-cell suppression and, upon their migration, to a pre-metastatic niche formation by Tregs in the firstline $\mathrm{CxCa}$ TDLN. Upon subsequent metastatic spread, monocytes are

Table 2 Theoretical advantages of low-dose, local immune potentiation in early stage cancer

\section{Low(er) tumor load}

2. Low(er) levels of immune suppression

3. Limited tumor heterogeneity: clonal neoantigens [58]

4. Systemic protection against distant recurrence [36, 37]

5. Single administration provides long-lasting protection [37]

6. Limited to no side effects [34, 35]

7. Pre-empts the need for expensive systemic therapies

8. Off-the-shelf generally applicable

9. Leveraging a (sub-optimally) primed T-cell repertoire in the TDLN [36] again recruited to the TME and converted into immunosuppressive M2-macrophages. These in turn expand and activate a new wave of Tregs that migrate to more distant TDLN and promote further metastatic spread through the LN catchment area. This vicious cycle of immune suppression may be interrupted by blocking the negative impact of PD-L1 ${ }^{+} \mathrm{M} 2$-like macrophages with intratumorally applied PD-L1 checkpoint blockade for an immune modulatory agent assumed to be primarily active in the tumor microenvironment [56]. Fransen et al. recently confirmed these results and, importantly, showed equal in vivo anti-tumor efficacy of low dose locally injected anti-PD-1 and of systemically administered high-dose antiPD-1 [57].

The rational design of future clinical trials targeting TDLN should encompass combinatorial use of immunotherapeutic agents, such as oncolytic viruses and/or immune checkpoint blocking antibodies. Moreover, it will likely not be limited to the cancer types discussed in this focused review, but may also be applied to other solid tumors proven amenable to immunotherapy, such as, e.g., lung cancer and head-and-neck cancer. 
Acknowledgements Graphical illustrations were drawn using the images from Servier Medical Art by Servier with slight modifications (http://www.smart.servier.com).

Author contributions TDG drafted the original outline of the review. JR, BDK, and AMH wrote the paper. TDG and ESJ added content and edited the paper. Final approval was given by all authors.

Funding Data discussed in this review were derived from studies funded by the Dutch Cancer Society (KWF Kankerbestrijding VU 2013-6015 and VU 2015-7864).

\section{Compliance with ethical standards}

Conflict of interest The authors declare that they have no conflicts of interest.

Ethical approval and ethical standards Not applicable.

Informed consent Not applicable.

Animal source Not applicable.

Cell line authentication Not applicable.

Open Access This article is distributed under the terms of the Creative Commons Attribution 4.0 International License (http://creativeco mmons.org/licenses/by/4.0/), which permits unrestricted use, distribution, and reproduction in any medium, provided you give appropriate credit to the original author(s) and the source, provide a link to the Creative Commons license, and indicate if changes were made.

\section{References}

1. Jones D, Pereira ER, Padera TP (2018) Growth and immune evasion of lymph node metastasis. Front Oncol 8:36

2. Farnsworth RH, Achen MG, Stacker SA (2018) The evolving role of lymphatics in cancer metastasis. Curr Opin Immunol 53:64-73

3. Zaal A, Zweemer RP, Zikán M, Dusek L, Querleu D, Lécuru F et al (2014) Pelvic lymphadenectomy improves survival in patients with cervical cancer with low-volume disease in the sentinel node: a retrospective multicenter cohort study. Int J Gynecol Cancer 24:303-311

4. Kenter GG, Hellebrekers BWJ, Zwinderman KH, De Vijver M, Van Peters LAW, Trimbos JB (2000) The case for completing the lymphadenectomy when positive lymph nodes are found during radical hysterectomy for cervical carcinoma. Acta Obs Gynecol Scand 79:72-76

5. Shah M, Lewin SN, Deutsch I, Burke WM, Sun X, Herzog TJ et al (2011) Therapeutic role of lymphadenectomy for cervical cancer. Cancer 117:310-317

6. D'Cruz AK, Vaish R, Kapre N, Dandekar M, Gupta S, Hawaldar $\mathrm{R}$ et al (2015) Elective versus therapeutic neck dissection in nodenegative oral cancer. N Engl J Med 373:521-529

7. Faries MB, Thompson JF, Cochran AJ, Andtbacka RH, Mozzillo $\mathrm{N}$, Zager JS et al (2017) Completion dissection or observation for sentinel-node metastasis in melanoma. N Engl J Med 376:2211-2222

8. Giuliano AE, Ballman KV, McCall L, Beitsch PD, Brennan MB, Kelemen PR et al (2017) Effect of axillary dissection vs no axillary dissection on 10-year overall survival among women with invasive breast cancer and sentinel node metastasis: The
ACOSOG Z0011 (Alliance) Randomized Clinical Trial. JAMA 318:918-926

9. Chen DS, Mellman I (2013) Oncology meets immunology: the cancer-immunity cycle. Immunity 39:1-10

10. Heusinkveld M, de Vos van Steenwijk PJ, Goedemans R, Ramwadhdoebe TH, Gorter A, Welters MJP et al (2011) M2 macrophages induced by prostaglandin E2 and IL-6 from cervical carcinoma are switched to activated M1 macrophages by $\mathrm{CD} 4^{+}$ Th1 cells. J Immunol. 187:1157-1165

11. Hazelbag S, Gorter A, Kenter GG, van den Broek L, Fleuren G (2002) Transforming growth factor- $\beta 1$ induces tumor stroma and reduces tumor infiltrate in cervical cancer. Hum Pathol 33:1193-1199

12. Kim MH, Seo SS, Song YS, Kang DH, Park IA, Kang SB et al (2003) Expression of cyclooxygenase-1 and -2 associated with expression of VEGF in primary cervical cancer and at metastatic lymph nodes. Gynecol Oncol 90:83-90

13. Karpanen T, Egeblad M, Karkkainen MJ, Kubo H, Yla-Herttuala S, Jaattela $\mathrm{M}$ et al (2001) Vascular endothelial growth factor C promotes tumor lymphangiogenesis and intralymphatic tumor growth. Cancer Res 61:1786-1790

14. van de Ven R, Lindenberg JJ, Oosterhoff D, de Gruijl TD (2013) Dendritic Cell plasticity in tumor-conditioned skin: CD14(+) cells at the cross-roads of immune activation and suppression. Front Immunol. 4:403

15. Swartz MA, Lund AW (2012) Lymphatic and interstitial flow in the tumour microenvironment: linking mechanobiology with immunity. Nat Rev Cancer 12:210-219

16. Chen J, Wang L, Yao Q, Ling R, Li K, Wang H (2004) Drug concentrations in axillary lymph nodes after lymphatic chemotherapy on patients with breast cancer. Breast Cancer Res 6:R474-R477

17. Elliott B, Cook MG, John RJ, Powell BWEM, Pandha H, Dalgleish AG (2004) Successful live cell harvest from bisected sentinel lymph nodes research report. J Immunol Methods 291:71-78

18. Vuylsteke RJCLM, van Leeuwen PAM, Meijer S, Wijnands PGJTB, Statius Muller MG, Busch DH et al (2002) Sampling tumor-draining lymph nodes for phenotypic and functional analysis of dendritic cells and T cells. Am J Pathol 161:19-26

19. Heeren AM, van Dijk I, Berry DRAI, Khelil M, Ferns D, Kole J et al (2018) Indoleamine 2,3-dioxygenase expression pattern in the tumor microenvironment predicts clinical outcome in early stage cervical cancer. Front Immunol 9:1598

20. Ferns DM, Heeren AM, Samuels S, Bleeker MCG, de Gruijl TD, Kenter GG et al (2016) Classical and non-classical HLA class I aberrations in primary cervical squamous- and adenocarcinomas and paired lymph node metastases. J Immunother Cancer 4:78

21. Heeren AM, de Boer E, Bleeker MCG, Musters RJP, Buist MR, Kenter GG et al (2015) Nodal metastasis in cervical cancer occurs in clearly delineated fields of immune suppression in the pelvic lymph catchment area. Oncotarget 6:32484-32493

22. Cochran AJ, Morton DL, Stern S, Lana AMA, Essner R, Wen D-R (2001) Sentinel lymph nodes show profound downregulation of antigen-presenting cells of the paracortex: implications for tumor biology and treatment. Mod Pathol 14:604-608

23. Cochran AJ, Huang RR, Lee J, Itakura E, Leong SPL, Essner R (2006) Tumour-induced immune modulation of sentinel lymph nodes. Nat Rev Immunol 6:659-670

24. Van De Ven R, Van Den Hout MFCM, Lindenberg JJ, Sluijter BJR, Van Leeuwen PAM, Lougheed SM et al (2011) Characterization of four conventional dendritic cell subsets in human skin-draining lymph nodes in relation to T-cell activation. Blood 118:2502-2510

25. Segura E, Valladeau-Guilemond J, Donnadieu M-H, Sastre-Garau X, Soumelis V, Amigorena S (2012) Characterization of resident and migratory dendritic cells in human lymph nodes. J Exp Med 209:653-660 
26. Sluijter BJR, van den Hout MFCM, Koster BD, van Leeuwen PAM, Schneiders FL, van de Ven R et al (2015) Arming the melanoma sentinel lymph node through local administration of CpG-B and GM-CSF: recruitment and activation of BDCA3/CD141(+) dendritic cells and enhanced cross-presentation. Cancer Immunol Res 3:495-505

27. van den Hout MFCM, Koster BD, Sluijter BJR, Molenkamp BG, van de Ven R, van den Eertwegh AJM et al (2017) Melanoma sequentially suppresses different DC subsets in the sentinel lymph node, affecting disease spread and recurrence. Cancer Immunol Res 5:969-977

28. Vollmer J, Krieg AM (2009) Immunotherapeutic applications of CpG oligodeoxynucleotide TLR9 agonists. Adv Drug Deliv Rev 61:195-204

29. Gershenwald JE, Scolyer RA, Hess KR, Sondak VK, Long GV, Ross MI et al (2017) Melanoma staging: evidence-based changes in the American Joint Committee on Cancer eighth edition cancer staging manual. CA Cancer J Clin 67:472-492

30. Eggermont AMM, Chiarion-Sileni V, Grob J-J, Dummer R, Wolchok JD, Schmidt $\mathrm{H}$ et al (2016) Prolonged survival in stage III melanoma with ipilimumab adjuvant therapy. N Engl J Med 375:1845-1855

31. Eggermont AMM, Blank CU, Mandala M, Long GV, Atkinson V, Dalle $S$ et al (2018) Adjuvant pembrolizumab versus placebo in resected stage III melanoma. N Engl J Med 378:1789-1801

32. Long GV, Hauschild A, Santinami M, Atkinson V, Mandalà M, Chiarion-Sileni V et al (2017) Adjuvant dabrafenib plus trametinib in stage III BRAF-mutated melanoma. N Engl J Med 377:1813-1823

33. Weber J, Mandala M, Del Vecchio M, Gogas HJ, Arance AM, Cowey CL et al (2017) Adjuvant nivolumab versus ipilimumab in resected stage III or IV melanoma. N Engl J Med 377:1824-1835

34. Vuylsteke RJCLM, Molenkamp BG, Gietema HA, Van Leeuwen PAM, Wijnands PGJTB, Vos W et al (2004) Local administration of granulocyte/macrophage colony-stimulating factor increases the number and activation state of dendritic cells in the sentinel lymph node of early-stage melanoma. Cancer Res 64:8456-8460

35. Molenkamp BG, Van Leeuwen PAM, Meijer S, Sluijter BJR, Wijnands PGJTB, Baars A et al (2007) Intradermal CpG-B activates both plasmacytoid and myeloid dendritic cells in the sentinel lymph node of melanoma patients. Clin Cancer Res 13:2961-2969

36. Molenkamp BG, Sluijter BJR, Van Leeuwen PAM, Santegoets SJAM, Meijer S, Wijnands PGJTB et al (2008) Local administration of PF-3512676 CpG-B instigates tumor-specific $\mathrm{CD}^{+} \mathrm{T}$-cell reactivity in melanoma patients. Clin Cancer Res 14:4532-4542

37. Koster BD, van den Hout MFCM, Sluijter BJR, Molenkamp BG, Vuylsteke RJCLM, Baars A et al (2017) Local adjuvant treatment with low-dose $\mathrm{CpG}$-B offers durable protection against disease recurrence in clinical stage I-II melanoma: data from two randomized phase II trials. Clin Cancer Res 23:5679-5686

38. Bonnefoi H, Litière $\mathrm{S}$, Piccart $\mathrm{M}$, MacGrogan $\mathrm{G}$, Fumoleau $\mathrm{P}$, Brain E et al (2014) Pathological complete response after neoadjuvant chemotherapy is an independent predictive factor irrespective of simplified breast cancer intrinsic subtypes: a landmark and twostep approach analyses from the EORTC 10994/BIG 1-00 phase III trial. Ann Oncol 25:1128-1136

39. DeNardo DG, Brennan DJ, Rexhepaj E, Ruffell B, Shiao SL, Madden SF et al (2011) Leukocyte complexity predicts breast cancer survival and functionally regulates response to chemotherapy. Cancer Discov 1:54-67

40. Kroemer G, Galluzzi L, Kepp O, Zitvogel L (2013) Immunogenic cell death in cancer therapy. Annu Rev Immunol 31:51-72

41. Honkoop AH, Luykx-de Bakker SA, Hoekman K, Meyer S, Meyer OW, van Groeningen CJ et al (1999) Prolonged neoadjuvant chemotherapy with GM-CSF in locally advanced breast cancer. Oncologist 4:106-111

42. Pinedo H, Buter J, Luykx-de Bakker S, Pohlmann P, van Hensbergen Y, Heideman DA et al (2003) Extended neoadjuvant chemotherapy in locally advanced breast cancer combined with GM-CSF: effect on tumour-draining lymph node dendritic cells. Eur J Cancer 39:1061-1067

43. De Vos Van Steenwijk PJ, Heusinkveld M, Ramwadhdoebe TH, Löwik MJ, Van Der Hulst JM, Goedemans R et al (2010) An unexpectedly large polyclonal repertoire of HPV-specific T cells is poised for action in patients with cervical cancer. Cancer Res 70:2707-2717

44. Stevanović S, Pasetto A, Helman SR, Gartner JJ, Prickett TD, Howie B et al (2017) Landscape of immunogenic tumor antigens in successful immunotherapy of virally induced epithelial cancer. Science 356:200-205

45. Biewenga P, van der Velden J, Mol BWJ, Stalpers LJA, Schilthuis MS, van der Steeg JW et al (2011) Prognostic model for survival in patients with early stage cervical cancer. Cancer 117:768-776

46. Heeren AM, Koster BD, Samuels S, Ferns DM, Chondronasiou D, Kenter GG et al (2015) High and interrelated rates of PD$\mathrm{L}^{+}{ }^{+} \mathrm{CD} 14^{+}$antigen-presenting cells and regulatory $\mathrm{T}$ cells mark the microenvironment of metastatic lymph nodes from patients with cervical cancer. Cancer Immunol Res 3:48-58

47. Zijlmans HJ, Fleuren GJ, Baelde HJ, Eilers PH, Kenter GG, Gorter A (2006) The absence of CCL2 expression in cervical carcinoma is associated with increased survival and loss of heterozygosity at 17q11.2. J Pathol 208:507-517

48. Heeren AM, Kenter GG, Jordanova ES, de Gruij1 TD (2015) CD14+ macrophage-like cells as the linchpin of cervical cancer perpetrated immune suppression and early metastatic spread: a new therapeutic lead? Oncoimmunology 4:e1009296

49. Lindenberg JJ, Oosterhoff D, Sombroek CC, Lougheed SM, Hooijberg E, Stam AGM et al (2013) IL-10 conditioning of human skin affects the distribution of migratory dendritic cell subsets and functional T cell differentiation. PLoS One 8:e70237

50. (2018) Pembrolizumab OK'd for cervical cancer. Cancer Discov 8:904

51. Rotman J, Mom CH, Jordanova ES, de Gruijl TD, Kenter GG (2018) 'DURVIT': a phase-I trial of single low-dose durvalumab (Medi4736) intratumourally injected in cervical cancer: safety, toxicity and effect on the primary tumour- and lymph node microenvironment. BMC Cancer 18:888

52. Marabelle A, Tselikas L, de Baere T, Houot R (2017) Intratumoral immunotherapy: using the tumor as the remedy. Ann Oncol 28:xii33-xii43

53. Koster BD, de Gruijl TD, van den Eertwegh AJM (2015) Recent developments and future challenges in immune checkpoint inhibitory cancer treatment. Curr Opin Oncol 27:482-488

54. Andrews A (2015) Treating with checkpoint inhibitors-figure $\$ 1$ million per patient. Am Health Drug Benefits 8:9

55. Fransen MF, van der Sluis TC, Ossendorp F, Arens R, Melief CJM (2013) Controlled local delivery of CTLA-4 blocking antibody induces $\mathrm{CD}^{+}{ }^{+} \mathrm{T}$-cell-dependent tumor eradication and decreases risk of toxic side effects. Clin Cancer Res 19:5381-5389

56. Chamoto K, Chowdhury PS, Kumar A, Sonomura K, Matsuda F, Fagarasan S et al (2017) Mitochondrial activation chemicals synergize with surface receptor PD-1 blockade for T cell-dependent antitumor activity. Proc Natl Acad Sci 114:E761-E770

57. Fransen MF, Schoonderwoerd M, Knopf P, Camps MG, Hawinkels LJ, Kneilling M et al (2018) Tumor-draining lymph nodes are pivotal in PD-1/PD-L1 checkpoint therapy. JCI Insight 3:e124507

58. McGranahan N, Furness AJS, Rosenthal R, Ramskov S, Lyngaa R, Saini SK et al (2016) Clonal neoantigens elicit T cell immunoreactivity and sensitivity to immune checkpoint blockade. Science 351:1463-1469

Publisher's Note Springer Nature remains neutral with regard to jurisdictional claims in published maps and institutional affiliations. 